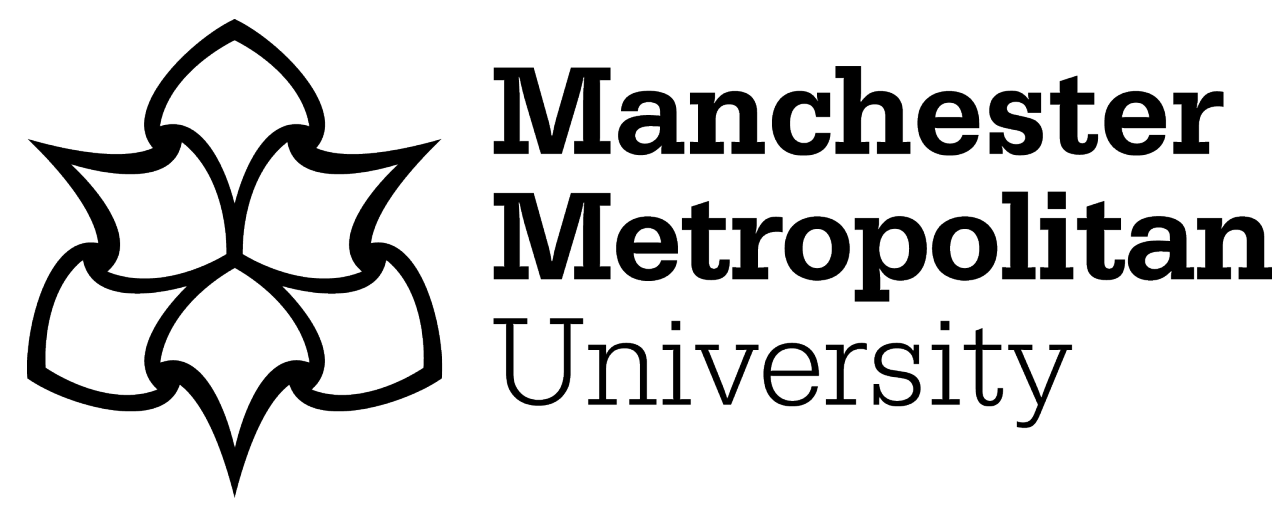

Djahel, Soufiene, Sommer, C and Marconi, A (2018) Guest Editorial: Introduction to the Special Issue on Advances in Smart and Green Transportation for Smart Cities. IEEE Transactions on Intelligent Transportation Systems, 19 (7). pp. 2152-2155. ISSN 1524-9050

Downloaded from: https://e-space.mmu.ac.uk/621291/

Version: Accepted Version

Publisher: IEEE

DOI: https://doi.org/10.1109/TITS.2018.2848018

Please cite the published version 


\title{
Guest Editorial: \\ Introduction to the Special Issue on Advances in Smart and Green Transportation for Smart Cities
}

\author{
Soufiene Djahel, Christoph Sommer and Annapaola Marconi
}

\begin{abstract}
According to a recent UN report, continuing population growth and urbanization are expected to increase the world's urban population by 2.5 billion people by 2050, with 2.9 billion extra vehicles. This massive growth in both population and number of vehicles, together with urban transformation and a trend towards mega cities, creates greater and more challenges for achieving smart transportation goals in smart cities. Therefore, new and more integrated modes of transportation, and environment friendly solutions are required to accommodate the rising demands of high liveability in smarter cities that offer safe, secure, affordable, reliable and sustainable transportation in old and new markets alike.
\end{abstract}

According to the EU commission, transportation accounts for more than one-fifth of the world's total $\mathrm{CO} 2$ emissions, the main greenhouse gas, from fossil fuel combustion, placing itself as a major contributor to global climate change. Moreover, the anticipated unprecedented increase in number of vehicles makes transportation a main target for reducing air pollution and achieving sustainable environment. All this incite public authorities and governments to devise new policies to reduce $\mathrm{CO} 2$ emissions by promoting public and green transport choices, supporting the private sector for inventing innovative modes of transport and stimulating a move towards cleaner and lower carbon vehicles. This ultimately leads to Green Transportation, which means any eco-friendly transport practice or vehicle that does not have any negative impact on the environment.

Within a Smart City, smart and green mobility has to be intended as the way in which citizens access and explore the city using advanced and eco-friendly transport modes. This implies being aware of the available mobility resources (public transports, parking facilities, bike lanes, ride sharing) and of their real value (in term of cost, time, carbon emissions, health), but also having an easy and efficient access to city services and events as well as a simple and unified access to transport payments. Supporting such an inter-connected, heterogeneous and dynamic transport system with lower or no environmental impact requires the adoption of innovative and sophisticated solutions from traffic management centers and city administration to control the mobility resources and policies and to proactively enhance them.

This special issue provides a collection of original highquality contributions presenting novel services, applications and solutions that help in reducing carbon emissions for any road transport mode or facilitate the access and use of modern and greener transport modes.
In total, we received 36 submissions. All papers were subject to the rigorous regular reviewing process of IEEE Transactions on Intelligent Transportation Systems. During the multi-stage reviewing process, 14 papers were accepted; from which 12 are included in this issue while the two remaining papers will appear in future regular issues.

The list of papers included in this special issue is given below.

Multi-Area Self-Adaptive Pricing Control in Smart City with EV User Participation

Yongquan Nie, Xiaolin Wang, and Ka Wai Eric Cheng

Promoting the market of Electric Vehicles (EV) can be one of the most effective ways to deal with the increasing severity of air pollution. However, the behavior of EV users can be rather stochastic and the aggregated charging power may add pressure to urban power grid during peak hours. In this paper, a novel smart city modeling with combined EV traveling and charging network is formulated. To alleviate the potential contingency brought by stochastic EV charging, mandatory control from grid operator and different kinds of electricity pricing schemes are introduced. Finally, the comparison between existing EV charging control schemes and multi-area self-adaptive (MASA) pricing control is performed to point out the limitation of passive control imposed on users as well as the flexibility of MASA pricing scheme. The results demonstrate that MASA pricing with active EV participation serves as an effective and economical solution to the future smart city under complex transportation network and massive EV integration.

Mobility-Aware Vehicle-to-Grid Control Algorithm in Microgrids

Haneul Ko, Sangheon Pack, and Victor Leung

To achieve the optimal performance in V2G system, a mobility-aware V2G control algorithm (MACA) that determines charging and discharging schedules for electric vehicles (EVs) is developed. The optimal policy on charging and discharging is obtained by a Markov decision process (MDP) with the consideration of the mobility of EVs, states of charge (SOC) of EVs, and the estimated/actual demands of microgrids (MGs). Since the mobility of EVs and the estimated/actual demand profiles of MGs may not be easily obtained, a reinforcement learning (RL) approach is also introduced. Evaluation results demonstrate that MACA with the optimal and learning-based policies can effectively achieve MG autonomy and provide higher satisfaction on the charging. 
Autonomous Vehicle Logistic System: Joint Routing and Charging Strategy

James J.Q. Yu and Albert Lam

The smart city embraces gradual adoption of autonomous vehicles $(\mathrm{AVs})$ into the intelligent transportation system. With full-fledged controllability, AVs can respond to instantaneous situations with high efficiency and flexibility. The authors propose a novel AV logistic system (AVLS) to accommodate logistic demands for smart cities. They focus on determining the optimal routes for the governed AVs in consideration of various requirements imposed by the vehicles, logistic requests, renewable generations, and the underlying transportation system. By coordinating their routes and charging schedules, the system can effectively utilize the renewable energy generated by distributed generations. The authors formulate the joint routing and charging problem in the form of quadratic-constrained mixed integer linear program. To improve its scalability, they developed a distributed solution method via dual decomposition. They conducted extensive simulations to evaluate the performance of the proposed system and the results show that AVLS can effectively utilize excessive renewable energy while accomplishing all logistic requests.

A Novel Infrastructure-based Worm Spreading Countermeasure for Vehicular Networks

Azzedine Boukerche and Qi Zhang

VANETs are essential components of intelligent transportation systems and are attracting an increasing amount of interest in research and industrial sectors. As multifunctional mobile nodes that integrate transporting, sensing, information processing, and wireless communication capabilities, vehicular nodes are facing remarkable security issues and are more vulnerable to malware attack than conventional communication nodes. In this paper, the authors examine the behaviors and security issues of worm spreading in VANETs. They discuss various approaches for worm spreading in VANETs and propose an infrastructure-based worm containment strategy. The infrastructure-based worm containment problem is modeled as a minimum contamination problem by introducing the expected contamination degree. The Simplified Greedy Method is then proposed to solve the minimum expected contamination degree problem on road networks. Simulation results show that the proposed method outperforms the existing greedy method and the max-flow based method from both complexity and solution quality aspects.

CreditCoin: A Privacy-Preserving Blockchain-based Incentive Announcement Network for Communications of Smart Vehicles

Lun Li, Jiqiang Liu, Lichen Cheng, Shuo Qiu, Wei Wang, Xiangliang Zhang, and Zonghua Zhang

An effective announcement network is proposed called CreditCoin, a novel privacy-preserving incentive announcement network based on Blockchain via an efficient anonymous vehicular announcement aggregation protocol. On the one hand, CreditCoin allows nondeterministic different signers (i.e., users) to generate the signatures and to send announcements anonymously in the non-fully trusted environment. On the other hand, with Blockchain, CreditCoin motivates users with incentives to share traffic information. The transactions and account information in CreditCoin can be traced by Trace Managers for malicious users' identities while they still achieve anonymity for others. CreditCoin thus is able to motivate users to forward announcements anonymously and reliably. Extensive experimental results show that CreditCoin is efficient and practical in simulations of smart transportation.

\section{Force-Driven Traffic Simulation for Future Connected} Autonomous Vehicle-Enabled Smart Transportation System Yang

Yuming Zhang, Guohui Zhang, Rafael Fierro, and Yin

A new continuum traffic flow dynamics model is developed for future Connected Autonomous Vehicle (CAV)enabled traffic systems by encapsulating mutually-coupled vehicle interactions using virtual internal and external forces. A Smoothed Particle Hydrodynamics (SPH)-based numerical simulation and an interactive traffic visualization framework are developed. This new model naturally preserves traffic volumes and automatically handles both longitudinal and lateral traffic operations due to its two-dimensional nature. The experiment shows that the developed model describes CAV traffic operations reasonably well and complements and generalizes the existing traffic flow models.

Detecting On-Street Parking Spaces in Smart Cities: Performance Evaluation of Fixed and Mobile Sensing Systems

Cristian Roman, Ruizhi Liao, Peter Ball, Shumao Ou, and Martin de Heaver

A mobile on street parking space detection system is presented. Drive tests have been carried out in an urban region where the available parking bays have fixed sensors embedded in each bay and the data collected from the mobile system has been compared to the corresponding fixed sensor data. The paper shows that by including map matching the accuracy of the GPS location information can be improved so that the performance of the mobile detection system approaches that of the fixed sensor system, whilst requiring significantly fewer sensor units.

High-Throughput Infrastructure for Advanced ITS Services: A Case Study on Air Pollution Monitoring

Jose Cecilia, Isabel Timon, Jesus Soto, Jose Santa, Fernando Pereñiguez, and Andres Muñoz

The authors of this paper introduce a high-throughput hardware-software infrastructure to provide novel ITS services efficiently, specially tailored to classification problems in Vehicle-to-Infrastructure (V2I). It relies on a fuzzy clustering technique that leverages heterogeneous computing servers based on CPU and several Graphics Processing Units (GPUs). The infrastructure is empirically tested to offer a geolocated pollution information service through the periodical collection of both vehicle's position and status data. Thus, it is 
offered a helpful service that correctly identifies highly polluting traffic areas and drivers. The experimental results show good performance of the system under high loads in real ambitious deployments.

A stigmergy-based analysis of city hotspots to discover trends and anomalies in urban transportation usage

Antonio Alfeo, Mario Cimino, Sara Egidi, Bruno Lepri, and Gigliola Vaglini

A novel stigmergy-based approach to analyze urban positioning data is proposed. By employing stigmergy, the authors obtain the characterization of the spatiotemporal dynamics in data. This characterization is used to obtain an adaptive and general-purpose measure of similarity between different dynamics. As a case study, the authors analyzed the taxi trip data gathered in Manhattan from 2013 to 2015 in order to (i) identify high-density urban areas (i.e. taxi hotspots), (ii) analyze their activity over time, (iii) detect and measure the impact of anomalous dynamics. This anomaly measure can be used by policy-makers to evaluate the effect of the proposed policies and change them dynamically.

\section{A Network Tomography Approach for Traffic} Monitoring in Smart Cities

Ruoxi Zhang, Sara Newman, Marco Ortolani, and Simone Silvestri

Traffic monitoring is a key enabler for several planning and management activities of a Smart City. However, traditional techniques are often not cost efficient, flexible, and scalable. The authors of this paper propose an approach to traffic monitoring that does not rely on probe vehicles, nor requires vehicle localization through GPS. Conversely, it exploits just a limited number of cameras placed at road intersections to measure car end-to-end traveling times. They modeled the problem within the theoretical framework of network tomography in order to infer the traveling times of all individual road segments in the road network. They specifically dealt with the potential presence of noisy measurements, and the unpredictability of vehicles paths. Moreover, they addressed the issue of optimally placing the monitoring cameras in order to maximize coverage, while minimizing the inference error, and the overall cost. They provided extensive experimental assessment on the topology of downtown San Francisco, CA, using real measurements obtained through the Google Maps APIs, as well as on realistic synthetic networks. Their approach provides a very low error in estimating the traveling times of more than $95 \%$ of all roads even when as few as $20 \%$ of road intersections are equipped with cameras.

\footnotetext{
Bikeshare Pool Sizing for Bike-And-Ride Multimodal Transit

Guoming S. Keshav, Lukasz Golab, and Kui Wu

The problem of optimal bicycle pool sizing at the transit stations is addressed, considering a multimodal transportation system with a regularly-scheduled public transportation backbone and shared bicycles for the first and last mile. Two probabilistic techniques are presented that guarantee bicycle
}

availability, with high probability, but using a smaller bicycle pool: i) a transient-state analysis based on the difference of random variables and ii) a steady-state analysis based on the Engset model.

\section{Boosting Ride Sharing with Alternative Destinations} Vinicius CezarMonteiro de Lira, Chiara Renso, Raffaele Perego, Salvvatore Rinzivillo, and Valeria Cesario Times

People living in highly-populated cities increasingly suffer an impoverishment of their quality of life due to pollution and traffic congestion. With the objective of reducing the number of circulating vehicles, the authors investigate a novel approach to boost ride-sharing opportunities based on the knowledge of the human activities behind individual mobility demands. They observed that in many cases the activity motivating the use of a private car (e.g., going to a shopping mall) can be performed in many different places. Therefore, when there is the possibility of sharing a ride, people having a pro-environment behavior or interested in saving money can accept to fulfill their needs at an alternative destination. They thus propose ACTIVITY-BASED RIDE MATCHING (ABRM), an algorithm aimed at matching ride requests with ride offers possibly reaching alternative destinations where the intended activity can be performed. By analyzing two large mobility datasets extracted from a popular social network, the authors showed that their approach could largely impact urban mobility by resulting in an increase up to $54.69 \%$ of ridesharing opportunities with respect to a traditional destinationoriented approach. Due to the high number of ride possibilities found by ABRM they introduced and assessed a subsequent ranking step to provide the user with the top-k most relevant rides only. They discussed how ABRM parameters affect the fraction of car rides that can be saved and how the ranking function can be tuned to enforce pro-environment behaviors.

Soufiene Djahel, Guest Editor

School of Computing, Mathematics \& Digital Technology

Manchester Metropolitan University

Chester Street, Manchester, M1 5GD,

United Kingdom

Email: s.djahel@mmu.ac.uk

Christoph Sommer, Guest Editor

EIM-I CMS, Univ. Paderborn

Fürstenallee 11

33102 Paderborn, Germany

Email: sommer@ccs-labs.org

Annapaola Marconi, Guest Editor

FBK Trento

Via alla Cascata 56/c - Povo

38100 Trento - ITALY

Email: marconi@fbk.eu 